\title{
Recherches sur la couverture médicale: questions circonstancielles et questions de fond
}

Dans le monde des médias, les nouvelles sont le plus souvent conçues de manière à répondre à des questions circonstancielles du genre: «Qu'est-il arrivé, quand, où, à qui, pourquoi?» Les journalistes basent leur travail sur ce type d'approche. Dans nos recherches sur la fourniture des soins, nous nous interrogeons aussi dans ce sens: «Combien de médecins y a-t-il?», «De quels médecins s'agit-il, où travaillent-ils, que font-ils?» et nous tentons de répondre à ces interrogations à l'aide des données dont nous disposons.

Les débats actuels sur le système de santé suisse débouchent immanquablement sur la question de l'insuffisance des données disponibles, qui rend la planification difficile. Qu'il s'agisse de Managed care, du nombre de médecins de premier recours, de l'offre et de la demande en prestations ou du thème de la qualité, on finit toujours par entendre qu'en Suisse, les bases de données de référence sont lacunaires et devraient être améliorées. On reproche aux études d'aboutir à des résultats trompeurs. Les chercheurs s'efforcent donc d'optimiser le réservoir d'informations à disposition, de réunir plusieurs séries de données, de montrer clairement les limites de ces dernières, et prennent diverses mesures pour augmenter la qualité de leurs analyses. La FMH participe elle aussi à l'amélioration des bases de données et aux analyses approfondies portant sur les champs de recherche existants et à venir. Elle a lancé un projet visant à actualiser ses propres données sur le corps médical suisse, de façon à pouvoir traiter plus fiablement encore les questions qui se posent actuellement et se poseront à l'avenir. La FMH contribue également à l'approfondissement des analyses sur la situation de la couverture médicale en Suisse, en fournissant par exemple à diverses institutions reconnues des données sur le corps médical suisse pour des projets d'envergure nationale.
Toutefois, les statistiques ne permettent pas de résoudre sans autre les problèmes de la santé publique. On prendra pour exemple la représentation de l'augmentation du nombre de médecins souvent citée par la presse ces derniers mois, représentation qui s'appuie sur la statistique médicale. Si ces chiffres sont publiés, il s'ensuit immédiatement - à part la discussion sur la qualité des données évoquée plus haut - une série de questions du genre:

- L'augmentation du nombre de médecins a-t-elle fait grimper les coûts?

- Le gel des admissions à pratiquer n'est-il pas appliqué sérieusement?

- Quel travail (en termes de durée et contenu) les médecins fournissent-ils dans leur effectif actuel par rapport à l'effectif d'autrefois?

Autant de thématiques complexes qui méritent d'être étudiées sérieusement, mais dont l'analyse ne suffira pas non plus à maîtriser la situation. Les réponses obtenues ne nous mettront pas à l'abri des questions de fond, que nous devrons tôt ou tard aborder. Un exemple: «Quels seraient les besoins en rhumatologues en Engadine?» Les statistiques ne nous permettent guère pour l'instant de répondre à une demande de ce genre - est-ce parce qu'à l'instar des médias, chacun ne se préoccupe que de questions circonstancielles? Et oublie, ou refoule, les questions de fond qu'il faudrait poser, à savoir celles qui touchent aux besoins en soins. La recherche sur la couverture médicale pourra apporter des éléments de réponse, mais elle ne pourra pas le faire seule. Ce sont les partenaires de la santé publique, le monde politique et, en fin de compte, la société toute entière, qui doivent trouver ensemble les réponses à cette question essentielle du besoin.

Martina Hersperger, responsable du département DDQ 